\title{
Up-link Capacity Derivation for Ultra-Narrow-Band IoT Wireless Networks
}

\author{
Yuqi MO • Minh Tien DO • Claire \\ GOURSAUD · Jean Marie GORCE
}

Received: 6 February 2017 / Accepted: 24 May 2017

\begin{abstract}
Thanks to its low energy consumption and very long range (up to $50 \mathrm{~km}$ in free-space), ultra-narrow-band transmission (UNB) represents a promising alternative to classical technologies used in cellular networks to serve low-throughput wireless sensor networks (WSNs) and the Internet of things (IoT). In UNB, nodes access to the medium by selecting their frequency in a random and continuous way. This randomness leads to new behavior in the interference which has not been theoretically analyzed, when considering the pathloss of nodes randomly deployed around the receiver. In this paper, in order to quantify the system performance, we derive and exploit two theoretical expressions of the outage probability in a UNB based IoT network, accounting for both interference due to the spectral randomness and path loss due to the propagation (with and without Rayleigh fading). This enables us to estimate the network capacity as a function of the path-loss exponent, by determining the maximum number of simultaneous supported nodes. We highlight that the bandwidth should be chosen based on the propagation channel properties.
\end{abstract}

Keywords UNB · Random-FTMA · network performance · capacity · channel characteristics $\cdot$ IoT

\section{Introduction}

Nowadays, the Internet of Things (IoTs) network is considered by many industry experts and excited consumers as the next industrial revolution or the next Internet. Indeed, it is expected to create a new interface between the internet

Yuqi MO, Minh Tien DO, Claire GOURSAUD, Jean Marie GORCE

Univ Lyon, INSA Lyon, Inria,

CITI,

F-69621 Villeurbanne, France

E-mail: mo.yuqi@insa-lyon.fr; name.surname@insa-lyon.fr 
and the physical world leading to applications such as smart metering, smart vehicules, surveillance, etc.[1].

By 2020, some analysts predict up to 20 billion of communicating devices in the world [2]. Deployed objects collect and wirelessly transfer their information. One may note that most of these nodes have only a small amount of data to transfer. Thus, a single collecting base station can serve, from a capacity point of view, a high number of nodes. For low density deployments, this permits a coverage of tens of kilometers. Nonetheless, the majority of devices are battery-based. As battery care and maintenance should be avoided owing to the high number of nodes, low energy consumption is also a strong requirement for IoT. Therefore, the challenging problems of IoT networks are the connection of high number of nodes, the management of bursty access to the medium and wide radio coverage, while ensuring low-cost device and energy efficiency. To meet these specific demands of IoT networks, new technologies dedicated to LPWAN (Low Power Wide Area Network) have recently emerged on the market [3], such as: LoRa [4] (promoted by the LoRa Alliance), RPMA [5] (developed and exploited by Ingenu), and UNB (Ultra Narrow Band, developed and exploited by SigFox) [6]. Interestingly, two opposing approaches have been exploited to perform the targeted long range transmission. Spread spectrum is considered in LoRa and RPMA, while UNB permits to transmitting messages by using extremely narrow band signals (100-200 Hz, about one thousand times smaller than the whole channel bandwidth). One may note that the transmission is randomly located in a much larger band, whose typical range is $192 \mathrm{kHz}$. Key advantages of UNB are its simplicity and its more extensive coverage in practice than the others [3]. We thus focus on UNB in this paper due to its higher point-to-point transmission range.

The natural channel access scheme of UNB is known as Random-FTMA (Random Frequency and Time Multiple Access). Each node can access the channel at its will, in an unslotted random way, both in time and frequency domains [7]. Different from pure ALOHA, Random-FTMA has the dual randomness in time and frequency. On one hand, the advantage of this uncontrolled access scheme is that the channel reservation of transmission is saved. On the other hand, this randomness in spectrum and time does not prevent from potential endogenous interference. In contrast to traditional transmissions where channelization can be performed, there can be partial spectral conflicts in UNB based networks. This specificity of the interference level has been detailed and modeled in [8], while its impact on the system performance has been estimated in [9]. It was shown that the interference power depends on a rejection coefficient which varies as a function of the spacing between the carrier frequencies. However, these studies considered a perfect channel case.

In practice, the received signal is attenuated, especially for such long range transmissions. Path loss is the main contributor of the received signal power attenuation, which depends mainly on the distance between the transmitter and the receiver. Moreover, multiple paths of signals caused by all the obstacles and reflectors in the environment are inevitable. Thus the impact of fading has also to be taken into account. For a network where numerous nodes are 
randomly located, stochastic geometry has been developed as an analytic tool to model the interference, and to quantify the network performance [10]. For example, stochastic geometry has been used to analyze systems such as simple random access in ad hoc networks (ALOHA) [11], dense IEEE 802.11 networks [12], multi-tier and cognitive cellular wireless networks [13-15].

All these works on stochastic geometry are interesting, because they provide interference models for different kinds of networks. Nevertheless, as all these studies are based on channelization, they do not consider the interference caused by partial collisions in the frequency domain. A way to introduce this characteristic in the stochastic geometry model is to consider a marked spatial Poisson Point Process (PPP), where the mark models the residual proportion of interference which is perceived on the desired transmission. However, due to the shape of the rejection coefficient function, according to our very first calculations, such approach leads to intractable expressions.

We thus exploit in this paper two complementary approaches. We first derive an analytic expression of a UNB-based system performance, when considering both the attenuation effect and the actual specific behavior of the interference in the spectral domain. Secondly, we use the stochastic geometry model to also take into account the Rayleigh fading effect, but with a simplified interference model.

The rest of the paper is constructed as follows: Section 2 first details UNB specificity and stochastic geometry related works, then in Section 3 we model the network and state all the hypothesis. In Section 4, we derive theoretically the expressions of outage probability in UNB network for two approaches. Then, in Section 5, we validate our analysis and present numerical results in Section 6. Finally, Section 7 concludes this paper.

\section{Background and Related Works}

\subsection{Ultra Narrow Band communications specificity}

Ultra-narrow-band refers to the fact that each individual node occupies an extremely narrow frequency band to transmit its signal. This frequency band is significantly smaller than the bandwidth of the channel and is usually around a few hundred Hertz.

In any RF communication, the intrinsic limitation is the noise floor, which determines the possibility of detecting the signal in the monitored bandwidth. As the noise contribution is proportional to the signal bandwidth $\left(N_{0}=\kappa\right.$. $T \cdot B$, where $\kappa$ is the Boltzmann constant, $T$ is the noise temperature, and $B$ is the total bandwidth), the narrower the signal is, the lower the noise is. Consequently, as the individual band used by a single transmitting node is around a few hundred Hertz in UNB, the noise floor is extremely low (around : $-154 \mathrm{dBm}$ for $100 \mathrm{~Hz}$ ). Thus, UNB reception power sensitivity is very low. This makes it possible to increase the propagation range compared to a classical technology such as narrow, medium or wide-band systems. This advantage 
permits serving a huge number of nodes deployed in an exceptionally large area (but at very low bit rates).

Nevertheless, as evoked in the introduction, the main specificity of UNB is the important uncertainty of the carrier frequency positioning [9]. Indeed, the crystal oscillator frequency is known to drift hundreds of thousands of $\mathrm{Hz}$ soon after power-up due to temperature variations, chip aging, etc [16]. For example, for an operating frequency band of $868 \mathrm{MHz}$ and a typical oscillator jitter $0.5-2$ parts per million ( $\mathrm{ppm}$ ), the uncertainty of carrier frequency positioning would be between $434 \mathrm{~Hz}$ and $1736 \mathrm{~Hz}$, which is much bigger than the transmission band occupied by an individual signal. Meanwhile, currently available technologies even provide factoring components with a larger standard deviation in the range of $2-20 \mathrm{ppm}$, while state-of-the-art components reach at best $0.25 \mathrm{ppm}[16,17]$. Thus, no current component can provide a sufficiently exact carrier frequency to avoid overlapping of simultaneous signals transmitted on a common bandwidth. When the frequency uncertainty is larger than the signal bandwidth, the system is said to be UNB.

Improved modulations (VMSK, VMCK, Q-VMCF etc. $[18,19]$ ) with enhanced error correction have been proposed to reduce the carrier frequencies instability impact. But, they bring only a modest gain while relying on complex signal processing and high energy consumption for battery-limited powered nodes. These solutions are not suitable to fulfill the compactness, low cost and energy constraints of the sensor nodes. Thus, the classic and simple BPSK modulation is used in UNB communications disregarding the oscillator uncertainty.

Therefore, the random nature of the carrier frequency has to be taken into account to evaluate UNB performance. First, on the base-station side, the main issue for the base station is to accurately detect the carrier frequency of the transmitted messages included in the (large) monitored bandwidth. For each detected transmission, the base station can then extract the signal at the identified frequency and decode the packet. Such detection and estimation rely on efficient SDR algorithms designed to analyze the total band (from 12 $\mathrm{kHz}$ up to $1 \mathrm{MHz}$ ) as described in a SigFox patent [20]. These algorithms are currently deployed in SigFox's networks and their features do not fall within the scope of this paper. Secondly, the carrier frequency randomness leads to a specific interference behavior which is developed for a single interferer in Section 3.3.

\subsection{Interference estimation}

Once the interference from one node is known, we have to evaluate the aggregated interference power (AIP). As UNB operates in an unlicensed band, UNB transmissions have to co-exist with many other signals such as LoRa. However, in this work, we focus on endogenous interference only, and comes from the spectral randomness of UNB signals. 
To characterize it in large-scale wireless networks, the wireless network node positions are usually modeled by the well-known Poisson point process (PPP). In particular, [21-23, 10,24,25] studied a large variety of networks including cellular networks, mobile ad-hoc and sensor networks. Stochastic geometry is an efficient tool for studying the average behavior over many spatial realizations of a network whose nodes are placed according to some probability distribution. Other approaches rely on deterministic lattices $[26,27]$ where the geographical area (urban, rural, and downtown) and the population activity are taken into account to approach the real-world non-uniform and irregular traffic distributions.

However, for UNB systems, PPP integrates more efficiently than lattices the spatial stochastic nature of the nodes distribution in addition to the attenuation laws. More importantly, marked PPP [22] provides a means of coping with additional random properties such as shadowing or fading if they are not spatially correlated.

The above mentioned references focus on approximating the interference distribution of large-scale wireless networks by computing the Laplace transform of the interference following [21]. However, this Laplace transform usually is not available in closed-form. Thus, approximating the interference distribution using well-known distributions such as Gaussian, inverse Gaussian, gamma, and inverse gamma distribution is appealing. Indeed, different methods permit to approximate the distribution, e.g. with moments matching [28], with the central limit theorem [29-31] or with the cumulants-based method in [32]. These approaches mainly permit to obtain the first moments (mean and variance) of the AIP probability density function (PDF) or the cumulative density function (CDF). Hence, closed-form results are available for both interference term and signal-to-interference ratios (SIR) to determine the network performance. These results have been extended to the case of multi-frequency systems but only on adjacent channels (thus barely interfering) [33-38]. However, in UNB networks, carrier frequencies are randomly chosen in a continuous interval. This has not been considered in earlier work, to the best of our knowledge.

\section{Modeling and assumptions}

\subsection{Network topology}

We consider in this paper the uplink of a cellular network, where nodes communicate only with a single Base Station (BS). The BS covers a finite circular area with a known radius $r_{M}$ and gathers the information from nodes located inside. Inter-cell interference is therefore not explicitly considered for simplicity's sake. Nonetheless, this can be done by modifying the Gaussian additive noise strength distribution as done in [39]. Indeed, the total interference produced by all the nodes beyond a given distance tends to a Gaussian additive noise when this distance is large enough [40]. 


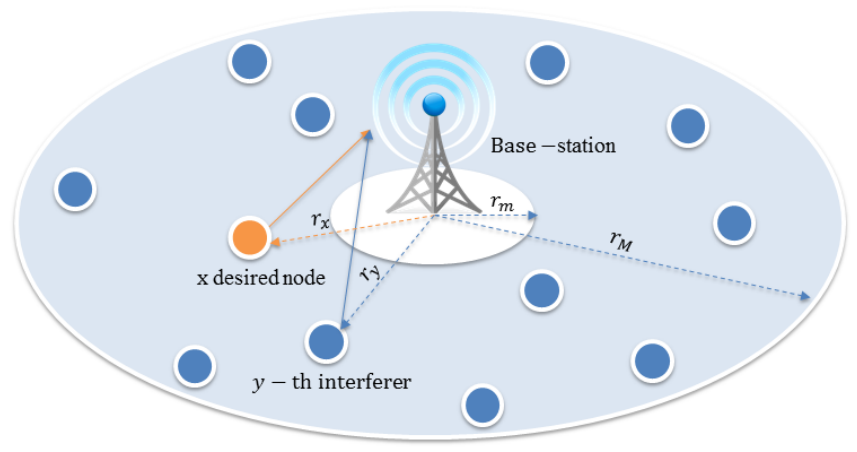

Fig. 1 Illustration of network topology: area range is $\left[r_{m}, r_{M}\right]$, orange point is the desired node, at a distance of $r_{x}$; blue point is interfering node, at a distance of $r_{y}$

We suppose that nodes are uniformly distributed in a disk form area, whose range is $\left[r_{m}, r_{M}\right]$, as shown in Fig. 1. Nodes are positioned inside the cell, except for the inner disk of the cell (to ensure mathematical tractability by avoiding singularity at the base-station location [24]). Nodes are distributed according to a spatial homogeneous Poisson point process (HPPP) of density $\lambda$ that lies in the Euclidean plan $\mathbb{R}^{2}$. We assume that nodes emit with the same emission power.

The BS is considered to be always in reception mode, and to scan the whole bandwidth for potential transmissions (as done in SigFox network). For each detected transmission (even simultaneous ones), the BS processes the incoming message(s).

\subsection{Medium Access}

The specificity of UNB medium access comes from the inherent oscillators' lack of precision. This prevents us from designing non-overlapping frequency channels with reasonable guard intervals [9]. It leads to a new paradigm for the multiple access scheme: Random-FTMA, and the interference modeling. We present in Fig. 2 an example of temporal and spectral repartition of nodes. With the randomness in both domains, we can have an overlap among nodes.

Indeed, contention mechanisms such as FTDMA or CSMA are not pertinent. While the first would require a centralized coordination to allocate slots to the nodes, the latter would require nodes to be able to sense the medium. Due to the very large cell size, the channel observation at nodes' point of view would be inefficient in avoiding collisions. Therefore, each node transmits without any knowledge on whether the chosen carrier frequency is currently used in the cell.

Besides, to avoid any traffic overload for synchronization, the proposed approach is not slotted in time. Consequently, the interference level may vary during the transmission of a given packet, as packets do not start (and stop) 
at the same time. The sleep or wake-up duty cycle may be different among different nodes. However, in order to simplify the analysis developed in this paper, this effect is not considered. The performance is based on instantaneous SINR distributions, to make sure that SINR is stable at the observed moment. We define $\mathcal{A}$ as the subset of active nodes at a given time $t=t_{0}$. The number of simultaneous active nodes $N$ thus depends on the number of nodes in the cell, the packet length (in time) and the transmission probability.

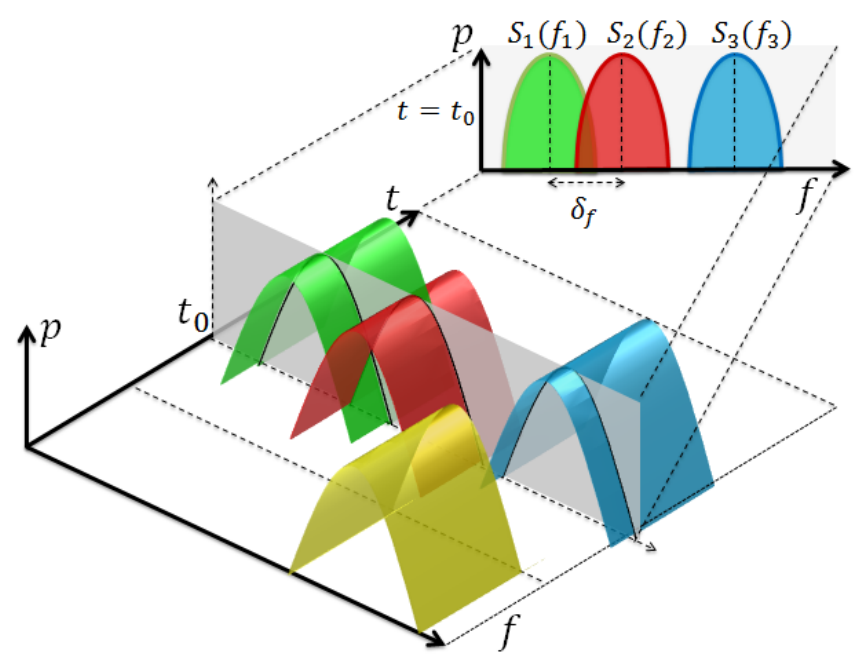

Fig. 2 Example of temporal \& spectral repartition of nodes

To sum up, from the receiver's point of view (i.e., on the base-station side), the monitored bandwidth contains a combination of narrow-band signals randomly located in time and frequency domains. This uncontrolled medium access may lead to interference or packet collisions among active nodes, especially when two or more nodes pick the same or close frequencies. This specific interference is described in the next section.

\subsection{Interference model for a single interferer}

Without loss of generality, we consider that node $x$ is the desired node, while all the others are called interfering nodes as they can collide with the desired node.

For a given transmission range $r$, the attenuation of the signal power received at the base-station follows

$$
h_{c}(r, t)=g(t) \cdot h_{0} \cdot r^{-\alpha}, r \in\left[r_{m}, r_{M}\right]
$$

where $\alpha \geq 2$ is the path loss exponent; $g$ is the Rayleigh channel coefficient, which is a random variable following an exponential distribution of unitary 
mean $g \sim \exp (1)$ considered as constant during the observation instant; and $h_{0}$ is the reference gain determined at the reference distance $r_{0}=1 \mathrm{~m}$.

Shadowing is neglected for the sake of simplicity but could be introduced with a marked PPP. It has been shown in [41] that a marked PPP with shadowing is equivalent to a PPP without shadowing by adapting the power strength in the model.

At the BS side, the received signal is the sum of active nodes' signals and can be expressed as follows:

$r(t)=\sqrt{h_{c}\left(r_{x}, t\right)} * h_{e}\left(f_{x}, t\right) * s_{x}(t)+\sum_{y \in\{\mathcal{A}-x\}} \sqrt{h_{c}\left(r_{y}, t\right)} * h_{e}\left(f_{y}, t\right) * s_{y}(t)+w(t)$

where, for any active node $x, y \in \mathcal{A}, s(t)$ is the modulated symbol; $h_{e}(f, t)$ is the transmission FIR filter centered on the randomly chosen carrier frequency $f$; $*$ denotes the convolution operator, and $w(t)$ is an additive white Gaussian noise with zero mean and variance $\sigma^{2}$.

To recover desired signal, the total signal received at BS is filtered at the carrier frequency of desired node $f_{x}$ with the matching filter $\left(h_{r}\left(f_{x}, t\right)=\right.$ $\left.h_{e}\left(f_{x}, t\right)\right)$, and can be written as follows:

$$
\begin{aligned}
r^{\prime}\left(f_{x}, t\right) & =h_{r}\left(f_{x}, t\right) * r(t) \\
& =\sqrt{h_{c}\left(r_{x}, t\right)} \cdot h_{e}\left(f_{x}, t\right) * h_{e}\left(f_{x}, t\right) * s_{x}(t) \\
& +\sum_{y \in\{\mathcal{A}-x\}} \sqrt{h_{c}\left(r_{y}, t\right)} \cdot h_{e}\left(f_{y}, t\right) * h_{e}\left(f_{x}, t\right) * s_{y}(t)+h_{e}\left(f_{x}, t\right) * w(t)
\end{aligned}
$$

We can first extract the received power corresponding to the signal of the desired node $x$ :

$$
P_{s}=h_{c}\left(r_{x}, t\right) \cdot P_{0}=g_{x} \cdot r_{x}^{-\alpha} \cdot P_{0}^{\prime}
$$

with $P_{0}=<\left|h_{e}(f, t) * h_{e}(f, t) * s(t)\right|^{2}>$ which is identical to all signals, and $P_{0}^{\prime}$ stands for $h_{0} \cdot P_{0}$.

Similarly, the interference power $I_{y}$ caused by a single interferer on the desired signal follows:

$$
I_{y}=h_{c}\left(r_{y}, t\right) \cdot \beta\left(\left|f_{x}-f_{y}\right|\right) \cdot P_{0}=g_{y} \cdot r_{y}^{-\alpha} \cdot \beta\left(\left|f_{x}-f_{y}\right|\right) \cdot P_{0}^{\prime}
$$

with $\beta\left(\left|f_{x}-f_{y}\right|\right)=\frac{\left.<\left|h_{e}\left(f_{y}, t\right) * h_{e}\left(f_{x}, t\right)\right|^{2}\right\rangle}{\left.<\left|h_{e}\left(f_{x}, t\right) * h_{e}\left(f_{x}, t\right)\right|^{2}\right\rangle}$ the rejection coefficient as the reception filter is centered on a different frequency than the transmission filter. This rejection coefficient quantifies the portion of interfering signal which is kept after filtering. It depends on the frequency spacing between the two carrier frequencies $\delta f=\left|f_{x}-f_{y}\right|$ as presented in Fig.3 (black curve) for a realistic filter as used in SigFox's network. We can observe that all the interference is kept (i.e. $\beta(\delta f)=1$ ) when $\delta f=0$ while it tends to 0 as the interferer frequency moves away from the desired node one. 
We can approximate this interference with two models. First, we can note that the actual interference can be divided into two main areas. Transition occurs between $200-400 \mathrm{~Hz}$, depending on the considered criterion. For high $\delta_{f}$, the interference level is low, below $-50 \mathrm{~dB}$. So a unique interferer in this area will have almost no impact on the performance of the desired node. On the contrary, a unique node will cause a perceptible interference only if $\delta_{f}$ is very small, as the filter is very selective. As the available bandwidth is very large (we consider at least $12 \mathrm{kHz}$ in this paper) compared to the interference width, the interference level can be approximated by a constant in this area. Thus, we model the contribution of a single-interferer by a rectangular function:

$$
\beta(\delta f)=\left\{\begin{array}{lll}
I_{\max } & \text { for } & \delta f \leq \triangle / 2, \\
I_{\min } & \text { for } & \delta f>\triangle / 2 .
\end{array}\right.
$$

where $\triangle$ corresponds to the width of $\delta_{f}$ that creates high interference level.

We can also approximate the interference power by a zero-mean Gaussian function, depending on the frequency difference $\delta f$ (pink curve on Fig.3):

$$
\beta(\delta f)=\frac{150}{\sigma \sqrt{2 \pi}} \exp ^{\frac{-\delta f^{2}}{2 \sigma^{2}}}
$$

with $\sigma=60[8]$.

Finally, one may note that when the carrier frequency of the desired signal is exactly located at the center of the total bandwidth this corresponds to the worst case. Indeed, at this frequency, the desired node suffers from statistically more interference than any other active node. Basically, for a fixed carrier frequency $f_{x}$ the frequency shifts $\delta_{f}$ are uniformly distributed in $\left[f_{\min }-f_{x} ; f_{\max }-f_{x}\right]$. Thus, for the central position, this interval is given by $[-B / 2 ; B / 2]$ while for a bottom-most carrier frequency, the interval is $[0 ; B]$. Consequently, assuming that the frequency response of the filter is monotonically decreasing, the center case is the worst case.

Fig.3 illustrates all the interference models. The black one represents the realistic UNB interference model, which is traced based on the SigFox network. The pink one represents the Gaussian function (7), which fairly approximates the realistic one, and will be used in section 4.1. The rectangles in green, red and blue represent respectively the approximated rectangular model (AR), the upper bound (UB) and the lower bound (LB). They will be used in Section 4.2.

\section{Theoretical analysis for UNB Networks in Spectral-Spatial Dimensions}

In this section, we derive the theoretical expression of the OP (Outage Probability) of any node $x$ located at $r_{x}$ from the base-station:

$$
O P=\mathbb{P}\left(\mathrm{SINR} \leq \gamma^{*}\right)=\mathbb{P}\left(\frac{P_{s}}{P_{I}+W} \leq \gamma^{*}\right)
$$



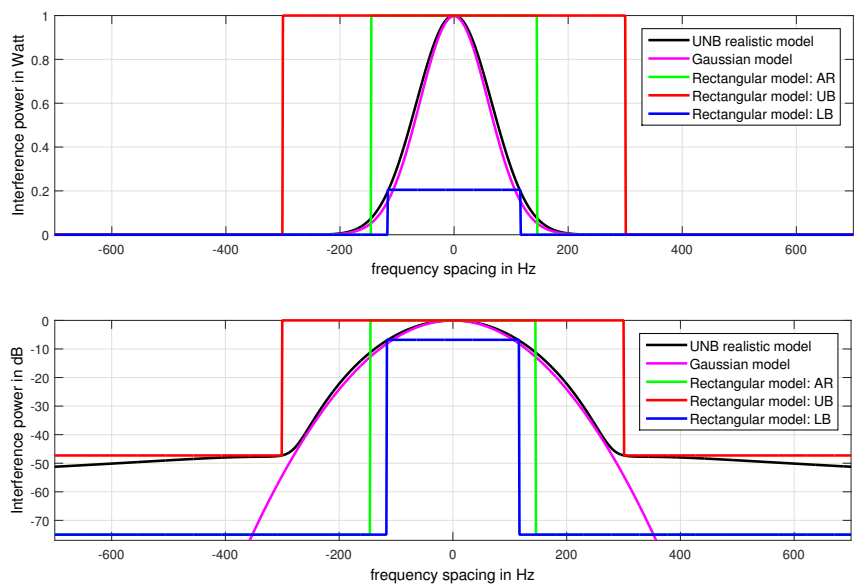

Fig. 3 Behavior of the interference coefficient vs the frequency difference $\delta_{f}$ between the desired node and the interferer

with $P_{s}$ the desired node received power (eq.(4)), $P_{I}$ the total interference power, $W$ the noise power and $\gamma^{*}$ the targeted SINR.

$P_{I}$ is obtained by summing the contribution of each interferer (5):

$$
P_{I}=\sum_{y \in\{\mathcal{A}-x\}} g_{y} \cdot r_{y}^{-\alpha} \cdot \beta(\delta f) \cdot P_{0}^{\prime}
$$

In this paper, the OP will be evaluated in 2 cases. We start with a simple scenario in 4.1, where we consider a simplified channel gain (no Rayleigh fading), and no noise. We then extend it to a more complete case in 4.2 , where we take into account Rayleigh fading and noise.

\subsection{Impact of path loss and approximated Gaussian model}

In order to have a first intuition of the random spectrum access impact on network performance, we first neglect fading and noise, and consider free space propagation $(\alpha=2)$. We derive the OP when considering path loss and spectral interference, by evaluating the SIR (Signal to Interference Ratio) as a simplification of the SINR. We use the approximated Gaussian model (pink curve in Fig.3) to measure the interference power. We focus on spectral interference coming from only one interferer, as this is the most frequent case according to actual SigFox network analysis. A more general case of the aggregation of several interferers will be treated in the second part.

Hence we have OP as:

$$
\mathbb{P}\left(\operatorname{SIR} \leq \gamma^{*}\right)=\mathbb{P}\left(\frac{P_{s}}{P_{I}} \leq \gamma^{*}\right)
$$


We first consider two active nodes (one desired node $x$, and one interfering node $y$ ) before generalizing it to $N$ active nodes. From (4) and (5), the SIR of the desired node can be expressed as:

$$
S I R=\frac{P_{s}}{P_{I}}=\frac{r_{x}^{-2} \cdot P_{0}^{\prime}}{r_{y}^{-2} \cdot \beta(\delta f) \cdot P_{0}^{\prime}}=\left(\frac{r_{y}}{r_{x}}\right)^{2} \frac{1}{\beta(\delta f)}
$$

A packet is considered lost if $S I R \leq \gamma^{*}$, i.e. when :

$$
r_{y} \leq r_{x} \sqrt{\gamma^{*} \beta(\delta f)}
$$

We decompose (12) by using the law of total probability with respect to the conditioning variable $\delta f$. Hence, the $O P$ becomes:

$$
\begin{aligned}
O P & =\int_{0}^{B} \mathbb{P}\left(S I R \leq \gamma^{*} \mid \delta f\right) \mathbb{P}(\delta f) \mathrm{d} \delta f \\
& =\int_{0}^{B} \mathbb{P}\left(r_{y} \leq r_{x} \sqrt{\gamma^{*} \beta(\delta f)} \mid \delta f\right) \mathbb{P}(\delta f) \mathrm{d} \delta f
\end{aligned}
$$

First of all, we express $\mathbb{P}(\delta f)$. Desired and interfering nodes choose their frequencies $f_{x}$ and $f_{y}$ randomly and uniformly in $[0, B]$. Thus, their spectral difference $\delta f$ follows the following probability distribution function:

$$
\mathbb{P}(\delta f)= \begin{cases}\frac{2}{B}\left(1-\frac{\delta f}{B}\right) & \text { for } \delta f \in[0, B] \\ 0 & \text { elsewhere }\end{cases}
$$

Secondly, we calculate $\mathbb{P}\left(r_{y} \leq r_{x} \sqrt{\gamma^{*} \beta(\delta f)} \mid \delta f\right)$.

Since nodes follow Poisson point process distribution, their respective distance to the BS follows an uniform distribution. $\mathbb{P}(r)$ represents the probability of being at a random distance (radius $r$ ) in a disk form area of $\left[r_{m}, r_{M}\right]$ :

$$
\mathbb{P}(r)=\left\{\begin{array}{lc}
\frac{2 r}{r_{M}^{2}-r_{m}^{2}}=\frac{2 r}{k^{2}} \text { for } r \in\left[r_{m}, r_{M}\right] \\
0 \quad \text { elsewhere }
\end{array}\right.
$$

with $k^{2}=r_{M}^{2}-r_{m}^{2}$. 
For a desired node at a given distance $r_{x}$, we can derive the conditional probability with respect to $\delta f$, according to different values of $r_{x} \sqrt{\gamma^{*} \beta(\delta f)}$ :

$$
\begin{aligned}
& \mathbb{P}\left(r_{y} \leq r_{x} \sqrt{\gamma^{*} \beta(\delta f)} \mid \delta f\right) \\
& = \begin{cases}\int_{r_{m}}^{r_{m}} \mathbb{P}\left(r_{y}\right) \mathrm{d} r_{y} & \text { if } r_{x} \sqrt{\gamma^{*} \beta(\delta f)} \leq r_{m}, \\
\int_{r_{m}}^{r_{x} \sqrt{\gamma^{*} \beta(\delta f)}} \mathbb{P}\left(r_{y}\right) \mathrm{d} r_{y} & \text { if } r_{m} \leq r_{x} \sqrt{\gamma^{*} \beta(\delta f)} \leq r_{M} \\
\int_{r_{m}}^{r_{M}} \mathbb{P}\left(r_{y}\right) \mathrm{d} r_{y} & \text { if } r_{x} \sqrt{\gamma^{*} \beta(\delta f)} \geq r_{M}\end{cases} \\
& = \begin{cases}0 & \text { if } r_{x} \sqrt{\gamma^{*} \beta(\delta f)} \leq r_{m}, \\
\frac{r_{x}^{2} \gamma^{*} \beta(\delta f)-r_{m}^{2}}{k^{2}} & \text { if } r_{m} \leq r_{x} \sqrt{\gamma^{*} \beta(\delta f)} \leq r_{M} \\
1 & \text { if } r_{x} \sqrt{\gamma^{*} \beta(\delta f)} \geq r_{M}\end{cases}
\end{aligned}
$$

We update (13) according to (16), and replace $\beta(\delta f)$ by the zero-mean Gaussian function as shown in (7). After integration over $\delta f$, OP becomes:

$$
\begin{aligned}
O P= & \int_{0}^{B} \mathbb{P}\left(r_{y} \leq r_{x} \sqrt{\gamma^{*} \beta(\delta f)} \mid \delta f\right) \mathbb{P}(\delta f) \mathrm{d} \delta f \\
= & 0+\int_{b_{1}}^{b_{2}}\left(\frac{r_{x}^{2} \gamma^{*} \beta(\delta f)-r_{m}^{2}}{k^{2}}\right) \mathbb{P}(\delta f) \mathrm{d} \delta f+\int_{b_{0}}^{b_{1}} 1 \cdot \mathbb{P}(\delta f) \mathrm{d} \delta f \\
= & {\left[75 a \gamma^{*} \operatorname{erf}\left(\frac{\delta f}{\sqrt{2 \sigma^{2}}}\right)+\frac{150 a \gamma^{*} \sigma}{B \sqrt{2 \pi}} \exp \left(\frac{-\delta f^{2}}{2 \sigma^{2}}\right)+e \frac{\delta f^{2}}{2}-e B \delta f\right]_{b_{1}}^{b_{2}} } \\
& +\frac{B}{2}\left[\delta f-\frac{\delta f^{2}}{2 B}\right]_{b_{0}}^{b_{1}}
\end{aligned}
$$

with the following constants:

$$
\begin{aligned}
& d=\frac{2 r_{x}^{2}}{B k^{2}}, \quad \quad \quad=\frac{2 r_{m}^{2}}{B^{2} k^{2}}, \quad b_{0}=0 \\
& b_{1}=\min \left(\beta^{-1}\left(\left(\frac{r_{M}}{r_{x}}\right)^{2} \frac{1}{\gamma^{*}}\right), B\right), \quad b_{2}=\min \left(\beta^{-1}\left(\left(\frac{r_{m}}{r_{x}}\right)^{2} \frac{1}{\gamma^{*}}\right), B\right)
\end{aligned}
$$

The integral bounds $b_{0}, b_{1}$ and $b_{2}$ were obtained as follows. First, $r_{m} \leq$ $r_{x} \sqrt{\gamma^{*} \beta(\delta f)} \leq r_{M}$ is equivalent to $\frac{r_{m}}{r_{x}} \leq \sqrt{\gamma^{*} \beta(\delta f)} \leq \frac{r_{M}}{r_{x}}$, and it can be expressed as $\beta^{-1}\left(\left(\frac{r_{M}}{r_{x}}\right)^{2} \frac{1}{\gamma^{*}}\right) \leq \delta f \leq \beta^{-1}\left(\left(\frac{r_{m}}{r_{x}}\right)^{2} \frac{1}{\gamma^{*}}\right)$. As the range of $\delta f$ is $[0, B]$, we have to put an min function to constrain these integral bounds in case they exceed $B$. One may note that $\beta(\delta f) \in[0,1]$. Thus, $b_{1}$ exists only if $\left(\frac{r_{M}}{r_{x}}\right)^{2} \leq \gamma^{*}$. If not, $b_{1}$ would be null, and the last term of integral would be null too. 
The OP derived here concerns a given $r_{x}$. In practice, the distance of the desired node vary over the cell. A similar study deriving the mean PER for the whole cell is presented in [42] for interested readers.

(17) represents the OP when there are 2 active nodes. We now extend it to $N$ active nodes. In this case, any of the $N-1$ nodes (i.e. all nodes except the desired node) can be an interfering node. Accordingly, transmission success means that the desired node is not interrupted by any of $N-1$ nodes. Consequently, the outage probability is given by:

$$
O P_{(N)}=1-(1-O P)^{N-1}
$$

4.2 Joint Impact of Path-loss, Rayleigh fading and approximated rectangular model

In this section, we derive the OP for a more complete channel model. In addition to the geometric pathloss, Rayleigh fading and noise are considered. However, to obtain tractable expressions, the spectral interference power $\beta(\delta f)$ is now described by the rectangular model (e.g. green, red and blue curves in Fig.3) as presented in (6). The expression of $\beta(\delta f)$ becomes less complex when using the rectangular model. Nevertheless, as we can see in Fig.3, the rectangular models can not reflect all details of the realistic function.

The SINR expression exploited in this section is given by:

$$
\mathrm{SINR}=\frac{g_{x} \cdot r_{x}^{-\alpha} \cdot P_{0}^{\prime}}{P_{I}+W}
$$

where $W$ is the filtered additive white Gaussian noise power.

Thus, for any node $x$ located at $r_{x}$ from the BS, OP can be written:

$$
\mathrm{OP}=\mathbb{P}\left(g_{x} \leq \frac{\gamma^{*}\left(W+P_{I}\right)}{r_{x}^{-\alpha} \cdot P_{0}^{\prime}}\right)
$$

Since the AIP $P_{I}$ is itself a random variable, this probability can be expressed as follows:

$$
\mathrm{OP}=\mathbb{E}_{P_{I}}\left[\mathbb{P}\left(g_{x} \leq \frac{\gamma^{*}\left(W+P_{I}\right)}{r_{x}^{-\alpha} \cdot P_{0}^{\prime}} \mid P_{I}\right)\right]
$$

As $g_{x}$ represents the coefficient of Rayleigh fading, and follows an exponential distribution of unitary mean $g_{x} \sim \exp (1)$, we get:

$$
\begin{aligned}
\mathrm{OP} & =1-\mathbb{E}_{P_{I}}\left[\exp \left(-\frac{\gamma^{*}\left(W+P_{I}\right)}{r_{x}^{-\alpha} \cdot P_{0}^{\prime}}\right)\right] \\
& =1-\exp \left(\frac{-\gamma^{*} W}{r_{x}^{-\alpha} \cdot P_{0}^{\prime}}\right) \cdot \mathbb{E}_{P_{I}}\left[\exp \left(-\frac{\gamma^{*} P_{I}}{r_{x}^{-\alpha} \cdot P_{0}^{\prime}}\right)\right] \\
& =1-\exp (-W \cdot s) \cdot \mathbb{E}_{P_{I}}\left[\exp \left(-P_{I} \cdot s\right)\right]
\end{aligned}
$$


where $s=\frac{\gamma^{*}}{r_{x}^{-\alpha} \cdot P_{0}^{\prime}}$.

The second term is nothing but the Laplace transform of the AIP probability density function(PDF)

$$
\mathcal{L}_{P_{I}}(s)=\mathbb{E}_{P_{I}}\left[\exp \left(-P_{I} \cdot s\right)\right] .
$$

Then, (22) can be expressed as:

$$
\mathrm{OP}=1-\exp (-W \cdot s) \cdot \mathcal{L}_{P_{I}}(s)
$$

which establishes the relation between OP and the Laplace transform of the AIP's PDF. It is worth noting that this relation holds because a Rayleigh fading has been considered.

\subsubsection{Laplace transform of AIP}

This section aims at deriving an analytical expression of (23), by following the track of previous seminal works on stochastic geometry for wireless networks. We provide here steps leading to the final result. But, for a deeper understanding of the Laplace functional method, we refer interested readers to $[10,22]$ for the general background and to [43] for the first application of this method to cellular networks.

Active nodes are distributed independently and uniformly in a circular area, with a HPPP process of density $\lambda$. The Laplace functional derivation first relies on the fact that the AIP $P_{I}$ is given as a sum, with respect to the active nodes of the HPPP, of a unique function : $P_{I}=\sum_{\Phi} f\left(r_{y}\right)$, with:

$$
f\left(r_{y}\right)=I_{y}\left(r_{y}\right)=g_{y} \cdot r_{y}^{-\alpha} \cdot \beta(\delta f) \cdot P_{0}^{\prime} .
$$

$f\left(r_{y}\right)$ describes the random interference contribution $I_{y}$ of a non-desired node (9). It depends on the position of the considered node, and on two additional random variables $\beta(\delta f)$ and $g_{y}$. In our case, the rejection coefficient $\beta(\delta f)$ along with $g_{y}$ are not related to the node position and are generated independently to each other. Thus $g_{y} \cdot \beta(\delta f)$ forms a spatially non correlated and homogeneous random parameter, independent of $h\left(r_{y}\right)$, and acts as a mark on the HPPP.

In this case, the Laplace transform of the AIP pdf can be identified to the Laplace functional $\mathcal{L}_{\Phi}(f)[22]$ of a marked HPPP of intensity measure $\lambda$, with a random mark $M=g_{y} \cdot \beta(\delta f)$ given by:

$$
\mathcal{L}_{\Phi}(f)=\exp \left(-\int_{\mathbb{R}^{2}}\left(1-\mathbb{E}_{M}\left[e^{-f(x)}\right]\right) \lambda(d x)\right) .
$$

In the context of this paper, using (25) and (26), the Laplace functional of the AIP is expressed as:

$$
\mathcal{L}_{P_{I}}(s)=\mathcal{L}_{\Phi}(f)=\exp \left(-\int_{0}^{2 \pi} \int_{r_{m}}^{r_{M}}\left(1-\mathbb{E}_{g_{y}, \beta(\delta f)}\left[e^{-s \cdot I_{y}\left(r_{y}\right)}\right]\right) \lambda r_{y} d r_{y} d \theta\right),
$$


with $I_{y}\left(r_{y}\right)$ as recalled in $(25)$, and $\lambda$ being the node density in nodes $/ \mathrm{m}^{2}$. This latter is defined as the ratio between the average number of simultaneously active nodes $N$ and the circular area where nodes are deployed.

According to the moment generating function (MGF) of a variable following an exponential distribution [43], and as $g_{y}$ and $\beta(\delta f)$ are independent, we obtain:

$$
\mathcal{L}_{P_{I}}(s)=\exp \left(-2 \pi \lambda \int_{r_{m}}^{r_{M}}\left(1-\mathbb{E}_{\beta(\delta f)}\left[\frac{1}{1+s \cdot \beta(\delta f) \cdot r_{y}^{-\alpha} \cdot P_{0}^{\prime}}\right]\right) r_{y} d r_{y}\right) .
$$

By considering the rectangular model (6) defined in Section 3.3 for $\beta(\delta f)$, the rejection factor follows a Bernoulli distribution with probability $p=\frac{2 \triangle}{B}$. Thus, the expectation term in the integral part (28) can be rewritten as:

$$
\begin{aligned}
\mathbb{E}_{\beta(\delta f)} & {\left[\frac{1}{1+s \cdot \beta(\delta f) \cdot r_{y}^{-\alpha} \cdot P_{0}^{\prime}}\right] } \\
& =p \cdot \frac{1}{1+s \cdot I_{\max } \cdot r_{y}^{-\alpha} \cdot P_{0}^{\prime}}+(1-p) \cdot \frac{1}{1+s \cdot I_{\min } \cdot r_{y}^{-\alpha} \cdot P_{0}^{\prime}} \\
& =p \cdot \frac{1}{1+s \cdot b \cdot r_{y}^{-\alpha}}+(1-p) \cdot \frac{1}{1+s \cdot c \cdot r_{y}^{-\alpha}}
\end{aligned}
$$

where $b$ and $c$ are respectively defined by $b=I_{\max } \cdot P_{0}^{\prime}$ and $c=I_{\min } \cdot P_{0}^{\prime}$. Combining (28) and (29), the Laplace distribution of the interference can be derived as:

$$
\begin{aligned}
\mathcal{L}_{P_{I}}(s)= & \exp \left(-2 \pi \lambda \int_{r_{m}}^{r_{M}}\left(1-\frac{p}{1+s \cdot b \cdot r_{y}^{-\alpha}}-\frac{1-p}{1+s \cdot c \cdot r_{y}^{-\alpha}}\right) r_{y} d r_{y}\right) \\
= & \exp \left(-2 \pi \lambda(\underbrace{\int_{r_{m}}^{r_{M}} r_{y} d r_{y}}_{A}-\underbrace{\int_{r_{m}}^{r_{M}} \frac{p}{1+s \cdot b \cdot r_{y}^{-\alpha}} r_{y} d r_{y}}_{B(s)}\right. \\
& -\underbrace{\int_{r_{m}}^{r_{M}} \frac{1-p}{1+s \cdot c \cdot r_{y}^{-\alpha}} r_{y} d r_{y}}_{C(s)}))
\end{aligned}
$$

The integral $A$ can be analytically computed and expressed as below:

$$
A=\int_{r_{m}}^{r_{M}} r_{y} d r_{y}=\frac{r_{M}^{2}-r_{m}^{2}}{2}
$$




\subsubsection{OP final expression}

By using (30) and (24), we can compute OP, for a given target SINR, for any desired node $x$ determined by its distance $r_{x}$ from the base-station (i.e., path-loss), and involving Rayleigh fading effect:

$$
\begin{aligned}
\mathrm{OP} & =1-\exp (-W \cdot s) \cdot \mathcal{L}_{P_{I}}(s) \\
& =1-\exp (-W \cdot s) \cdot \exp (-2 \pi \lambda(A-B(s)-C(s)))
\end{aligned}
$$

with the constants $A, B(s)$ and $C(s)$ as shown in (30).

One may note that $B(s)$ and $C(s)$ can not be analytically expressed for the general case, but are numerically computable. Nonetheless, for specific value of $\alpha$, the integration is possible. We present in next part the expressions of OP for two cases of interest: $\alpha=2$ (free space propagation), and $\alpha=4$ (relatively lossy environments and for the case of full specular reflection from the earth surface).

\subsubsection{OP for free space $\alpha=2$}

When $\alpha=2$, we can compute $B(s)$ and $C(s)$ :

$$
B(s)=\int_{r_{m}}^{r_{M}} \frac{p}{1+s \cdot b \cdot r_{y}^{-2}} r_{y} d r_{y}=\frac{p}{2}\left(r_{M}^{2}-r_{m}^{2}\right)+\frac{p}{2} \cdot s \cdot b \cdot \ln \left(r_{M}^{2}-r_{m}^{2}\right)
$$

$C(s)=\int_{r_{m}}^{r_{M}} \frac{1-p}{1+s \cdot c \cdot r_{y}^{-2}} r_{y} d r_{y}=\frac{1-p}{2}\left(r_{M}^{2}-r_{m}^{2}\right)+\frac{1-p}{2} \cdot s \cdot c \cdot \ln \left(r_{M}^{2}-r_{m}^{2}\right)$

Combined with $A$ (31), we can have the Laplace function of AIP as:

$$
\mathcal{L}_{P_{I}}(s)=\left(\frac{r_{m}^{2}+s b}{r_{M}^{2}+s b}\right)^{\pi \lambda p s b} \cdot\left(\frac{r_{m}^{2}+s c}{r_{M}^{2}+s c}\right)^{\pi \lambda(1-p) s c}
$$

Then, OP can be obtained as:

$$
\begin{aligned}
\mathrm{OP} & =1-\exp (-s \cdot W) \cdot \mathcal{L}_{P_{I}}(s) \\
& =1-\exp (-s \cdot W) \cdot\left(\frac{r_{m}^{2}+s b}{r_{M}^{2}+s b}\right)^{\pi \lambda p s b} \cdot\left(\frac{r_{m}^{2}+s c}{r_{M}^{2}+s c}\right)^{\pi \lambda(1-p) s c}
\end{aligned}
$$

with $s=\frac{\gamma^{*}}{r_{x}^{-2} \cdot P_{0}^{\prime}}$. 
4.2.4 OP for flat earth model $\alpha=4$

Similarly, when $\alpha=4$, we can compute $B(s)$ and $C(s)$ as shown below:

$$
\begin{gathered}
B(s)=\int_{r_{m}}^{r_{M}} \frac{p}{1+s \cdot b \cdot r_{y}^{-4}} r_{y} d r_{y} \\
=\frac{p}{2}\left(r_{M}^{2}-r_{m}^{2}\right)+\frac{p}{2} \cdot \sqrt{s b} \cdot \arctan \left(\frac{r_{m}^{2} \sqrt{s b}-r_{M}^{2} \sqrt{s b}}{s b+r_{M}^{2} r_{m}^{2}}\right) \\
C(s)=\int_{r_{m}}^{r_{M}} \frac{1-p}{1+s \cdot c \cdot r_{y}^{-4}} r_{y} d r_{y} \\
=\frac{1-p}{2}\left(r_{M}^{2}-r_{m}^{2}\right)+\frac{1-p}{2} \cdot \sqrt{s c} \cdot \arctan \left(\frac{r_{m}^{2} \sqrt{s c}-r_{M}^{2} \sqrt{s c}}{s c+r_{M}^{2} r_{m}^{2}}\right)
\end{gathered}
$$

Combined with $A$ (31), we can derive the Laplace function of AIP as:

$$
\begin{aligned}
\mathcal{L}_{P_{I}}(s)=\exp & \left(\pi \lambda p \cdot \sqrt{s b} \cdot \arctan \left(\frac{r_{m}^{2} \sqrt{s b}-r_{M}^{2} \sqrt{s b}}{s b+r_{M}^{2} r_{m}^{2}}\right)\right. \\
& \left.+\pi \lambda(1-p) \cdot \sqrt{s c} \cdot \arctan \left(\frac{r_{m}^{2} \sqrt{s c}-r_{M}^{2} \sqrt{s c}}{s c+r_{M}^{2} r_{m}^{2}}\right)\right)
\end{aligned}
$$

Then, OP can be expressed as:

$$
\begin{aligned}
\mathrm{OP}=1 & -\exp (s \cdot W) \cdot \exp \left(\pi \lambda p \cdot \sqrt{s b} \cdot \arctan \left(\frac{r_{m}^{2} \sqrt{s b}-r_{M}^{2} \sqrt{s b}}{s b+r_{M}^{2} r_{m}^{2}}\right)\right. \\
& \left.+\pi \lambda(1-p) \cdot \sqrt{s c} \cdot \arctan \left(\frac{r_{m}^{2} \sqrt{s c}-r_{M}^{2} \sqrt{s c}}{s c+r_{M}^{2} r_{m}^{2}}\right)\right)
\end{aligned}
$$

with $s=\frac{\gamma^{*}}{r_{x}^{-4} \cdot P_{0}^{\prime}}$.

We will use this theoretical expression of OP to evaluate the network capacity in terms of maximum number of simultaneous nodes. Results are shown in the next section.

\section{Validation}

In this section, we compare simulation results to theoretical ones (18) and (32). In our simulation framework, transmissions are performed with the UNB modulation technique. Binary data are broadcast with a BPSK modulation at a very low rate $\left(R_{b}=100 \mathrm{bps}\right)$. The transmitted signal thus occupies a very narrow band (i.e. about $b=100 \mathrm{~Hz}$ ). Transmissions are realized at a randomly chosen carrier frequency in a much larger band $B$ (typically $192 \mathrm{kHz}$ ). We also 
target $\gamma^{*}=6.8 \mathrm{~dB}$, corresponding to a bit-error-rate of $10^{-3}$ with a BPSK modulation.

Simulations are performed with different behavior of the interference rejection coefficient. We consider the real shape of the UNB interference model (black curve on Fig. 3), as well as rectangular models. For the rectangular model, we consider three set of parameters, which correspond to an approximated rectangular model (AR, green curve on Fig. 3), an upper bound (UB, red curve on Fig. 3) and a lower bound (LB, blue curve on Fig. 3), whose values are:

$$
\begin{aligned}
& A R: \triangle=145 \mathrm{~Hz}, \quad I_{\max }=0 \mathrm{~dB}, \quad I_{\min }=-75 \mathrm{~dB} \\
& U B: \triangle=300 \mathrm{~Hz}, \quad I_{\max }=0 \mathrm{~dB}, \quad I_{\min }=-47.28 \mathrm{~dB} \\
& L B: \triangle=116 \mathrm{~Hz}, \quad I_{\max }=-6.8 \mathrm{~dB}, \quad I_{\min }=-75 \mathrm{~dB}
\end{aligned}
$$

We used the same method as in [8] to determine the AR model parameters. This model assures the accuracy for cases where the targeted SINR is $6.8 \mathrm{~dB}$ and the desired node is at the edge of the cell range. Indeed, we have identified that higher distance leads to a wider rectangle. Thus, $r_{x}=r_{\max }$ provides the most pessimistic rectangular model over the cell. Since the AR model also depends on $\gamma^{*}$, we introduce an UB and a LB of the rectangular model, to estimate the range of OP.

We present on Fig. 4-6 the comparison between simulation and theory results. OP is plotted as a function of the desired node's distance $r_{x}$, the node density $\lambda$, and the bandwidth $B$ respectively. On all these figures, we can note that for the no-Rayleigh fading case, realistic simulation results match well with Gaussian theoretical ones (18). Besides, for Rayleigh fading case, the OP obtained with the theoretical model (32) fits very well with the simulated results obtained with the associated rectangular model. Therefore, we can deduce that the formula (18) and (32), as well as the accuracy of the approximated Gaussian model, are validated.

In addition, the evolution of OP with realistic UNB filter (black dash curve) lies between the UB (red curves) and LB (blue curves). As for the AR model (green curves), it slightly overestimates the realistic simulations, which comes from the choice of this model's parameters. Nonetheless, when $r_{x}$ becomes larger, OP can be more accurately estimated by the AR model. Similar results were also obtained when varying other parameters such as $\gamma^{*}, r_{M}$ and $r_{m}$, but are not presented here for space constraints. Those results all confirm the pertinent choice of the AR model, UB and LB, and the scalability of the rectangular models.

\section{Numerical results and exploitation}

6.1 Numerical results analysis

Theories validation aside, we can draw further conclusions from Fig. 4-6. In Fig. 4, we can see that OP increases when $r_{x}$ increases. Indeed, when the de- 


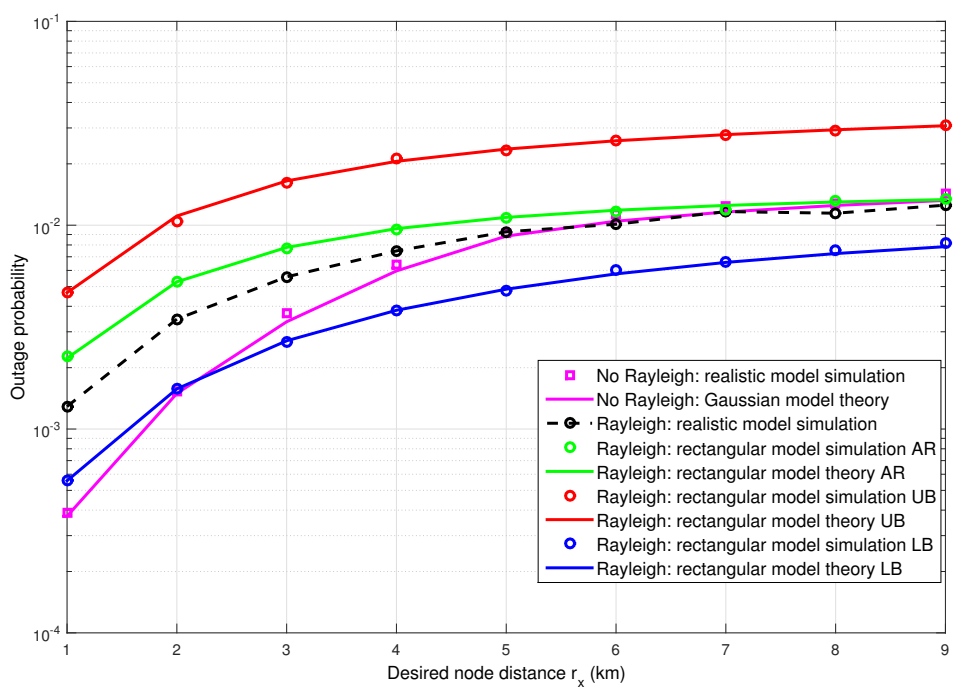

Fig. $4 \mathrm{OP}$ as a function of the desired node's distance $r_{x}$, for $B=96 \mathrm{kHz}, N=6, r_{M}=10$ $\mathrm{km}, r_{m}=1 \mathrm{~m}, \gamma^{*}=6.8 \mathrm{~dB}$ and path loss $\alpha=2$.

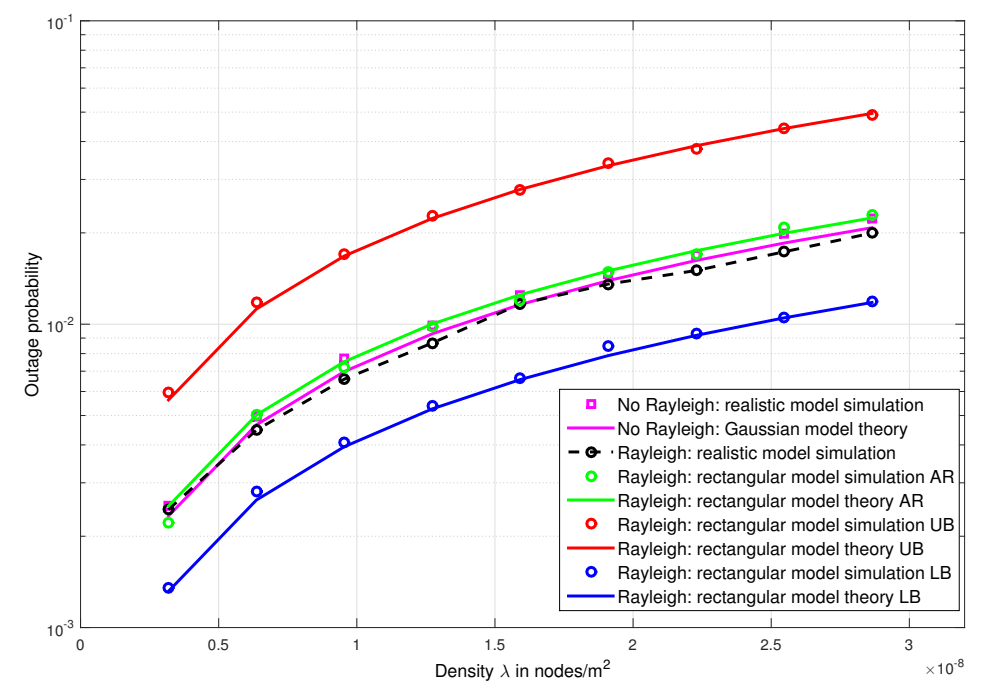

Fig. $5 \mathrm{OP}$ as a function of active nodes number $N$, for $B=96 \mathrm{kHz}, r_{x}=7 \mathrm{~km}, r_{M}=10$ $\mathrm{km}, r_{m}=1 \mathrm{~m}, \gamma^{*}=6.8 \mathrm{~dB}$ and path $\operatorname{loss} \alpha=2$.

sired node $x$ is further from BS, its received power is more attenuated because of path loss. This makes the desired signal more vulnerable to interferers. Interestingly, we can observe that OP(no-Rayleigh) is lower than OP(Rayleigh) when $r_{x}$ is small. But when $r_{x}$ exceeds a certain distance, these two cases have identical outage probabilities (and close to the real shape simulation results). 


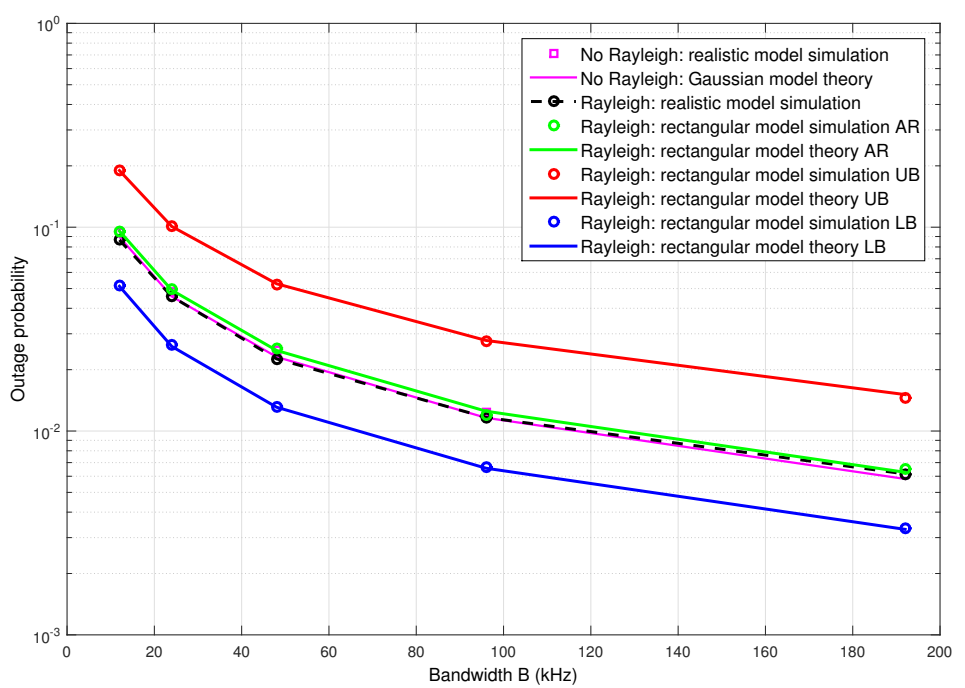

Fig. $6 \mathrm{OP}$ as a function of bandwidth $B$, for $N=6, r_{x}=7 \mathrm{~km}, r_{M}=10 \mathrm{~km}, r_{m}=1 \mathrm{~m}$, $\gamma^{*}=6.8 \mathrm{~dB}$ and path loss $\alpha=2$.

This phenomenon comes from the impacts of Rayleigh fading. Rayleigh fading can amplify or attenuate the received signal power. If the received power of the desired node gets attenuated, the area where interfering nodes may lead to error becomes larger. Hence we have more potential nodes in this case. Meanwhile, if the desired node's received power gets amplified, this area becomes smaller, which leads to less interfering nodes. When $r_{x}$ is small, the expected area's increase is much bigger than the expected area's decrease. Thus, such a node is sensitive to fading. On the contrary, for high $r_{x}$, the area increase is bounded by the cell limit, and thus is less important. In addition, for high $r_{x}$, the inner interferers are not impacted by fading in average. Hence, such node is barely affected by the fading.

In Fig. 5-6, we have fixed the desired node's distance so that the same performance is obtained without and with Rayleigh fading. We can see that OP increases with the node density $\lambda$, or as the bandwidth $B$ decreases. Indeed, as there are more nodes transmitting at the same time, or when the available transmission resources becomes smaller, the chance that their frequencies fall into the interfering zone gets higher. More interestingly, the gap between $\mathrm{OP}$ (no-Rayleigh) and $\mathrm{OP}$ (Rayleigh) is not influenced by $\lambda$ or $B$. Hence (18) can be used for high $r_{x}$ cases, whereas (32) is needed for low $r_{x}$ ones.

\subsection{Exploitation: Estimation of the network capacity}

In this section, we exploit the OP theoretical expressions to find out the maximum capacity. This parameter is defined in this study by the maximum number of active nodes $N$ that the network can support simultaneously, while 


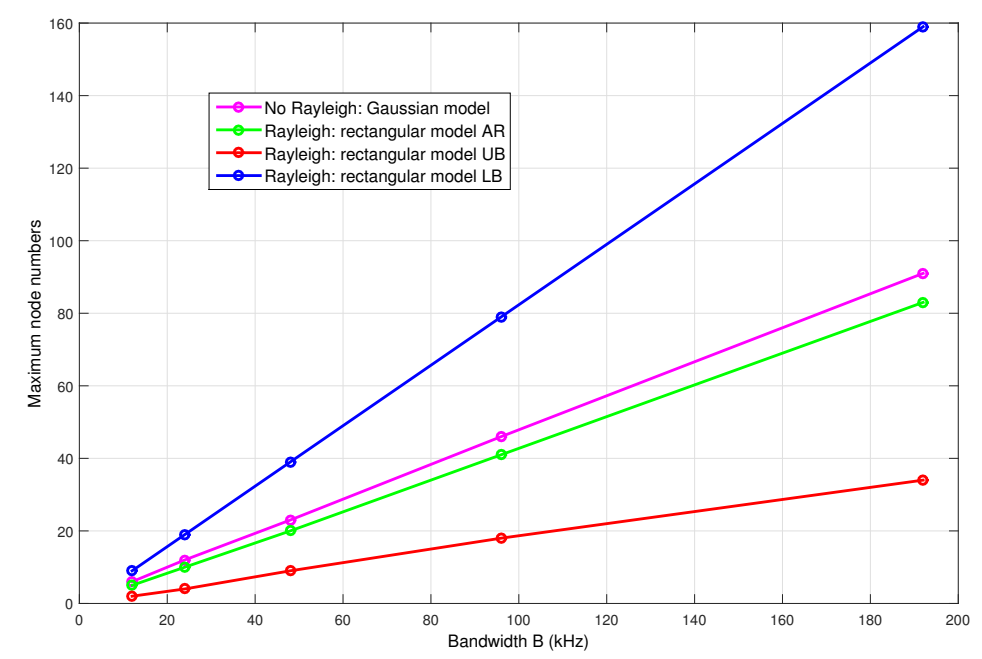

Fig. 7 Maximum node number vs bandwidth $B$, for targeted $O P=0.1, r_{x}=7 \mathrm{~km}$, $r_{M}=10 \mathrm{~km}, r_{m}=1 \mathrm{~m}, \gamma^{*}=6.8 \mathrm{~dB}$ and path loss $\alpha=2$.

maintaining a given OP constraint. We have reported on Fig.7-9 the capacity for a targeted $\mathrm{OP}=10^{-1}$, by considering the no-Rayleigh fading case (18); and the Rayleigh fading case (32). Capacities estimated by the UB and LB provide us the reliable range of the maximum active nodes while the AR model provides a good estimation, though not exact.

In Fig.7, we can observe that the capacity increases almost linearly with the available bandwidth. Indeed, as $B$ increases, we obtain a higher capacity thanks to less interference in the frequency domains. It is thus easy to scale the bandwidth in accordance to the number of nodes to serve, when keeping the same targeted OP.

In Fig.8, we can note that the overall capacity decreases when $r_{x}$ increases. As the desired node increasingly moves away from the BS, its received signal gets strongly attenuated. Hence, in order to successfully detect the desired node, the maximum node number has to be degraded.

Similarly, in Fig.9, we vary the cell radius $r_{M}$. As the cell size increases, the overall capacity increases. Indeed, when the cell surface becomes larger, by keeping $r_{x}$ unchanged, the desired node is perceived closer to BS than other nodes. This makes the desired node's signal easier to be detected. Thus the maximum node number increases for the targeted OP.

Finally, for a more fair comparison of the effective use of the bandwidth, we consider now the spectral efficiency. This term is determined as the ratio of the maximum node number and the bandwidth $\frac{N_{\max }}{B}$. We compare the spectral efficiency in two cases : without and with a guard band, for different values of $\alpha$, as shown in Fig.10. The without guard band corresponds to the ideal case, where targeted frequencies can be obtained, thus allowing the frequency random selection on the whole $B$. However, in practice, we could 


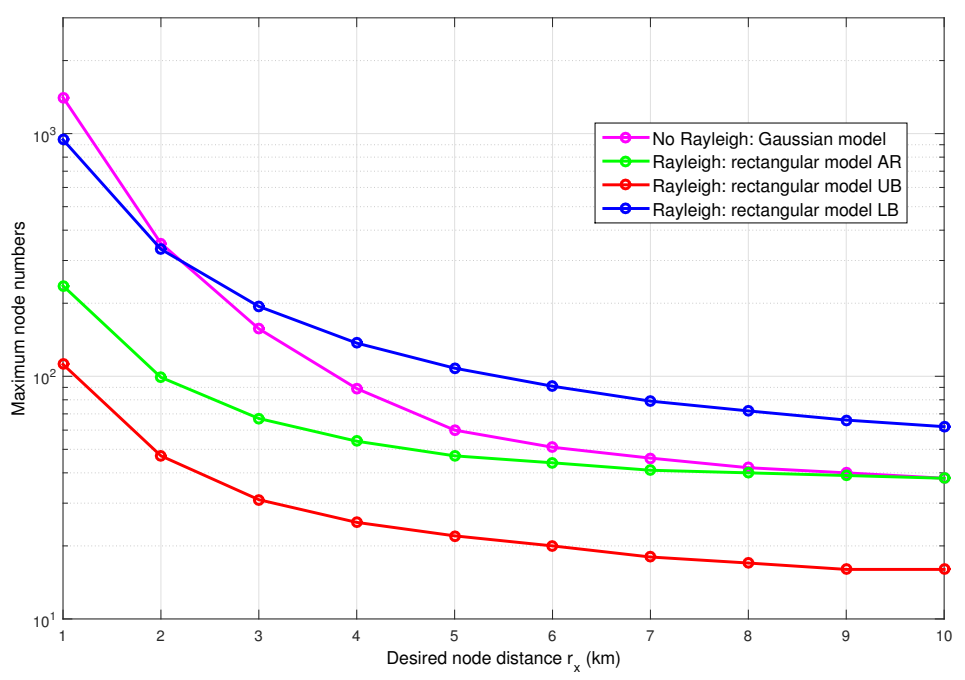

Fig. 8 Maximum node number vs desired node distance $r_{x}$, for targeted $O P=0.1, B=96$ $\mathrm{kHz}, r_{M}=10 \mathrm{~km}, r_{m}=1 \mathrm{~m}, \gamma^{*}=6.8 \mathrm{~dB}$ and path loss $\alpha=2$.

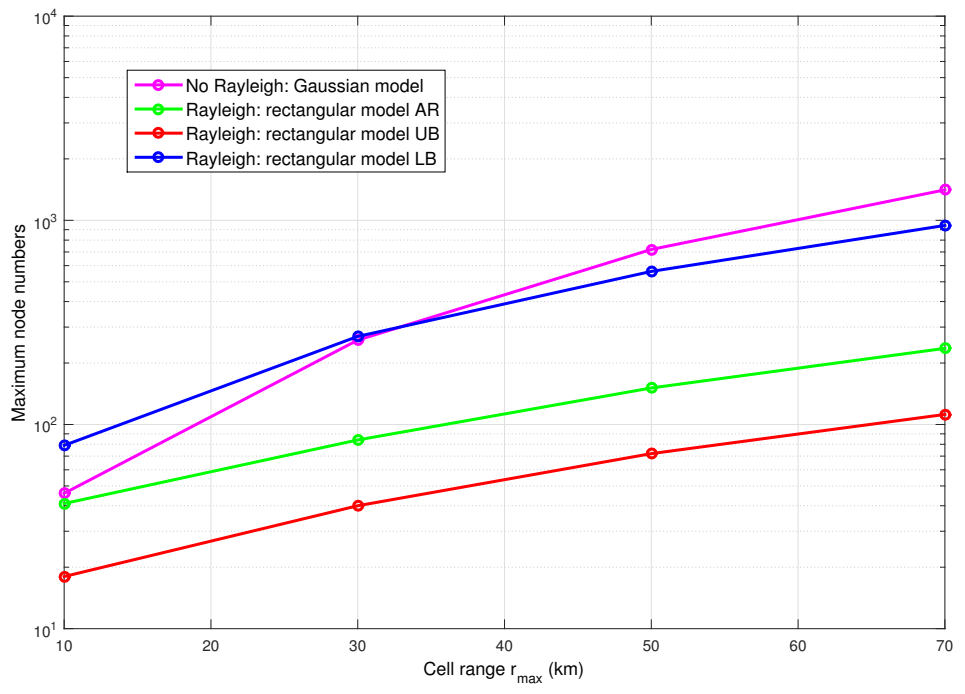

Fig. 9 Maximum node number vs cell range $r_{M}$, for targeted $O P=0.1, B=96 \mathrm{kHz}$, $r_{x}=7 \mathrm{~km}, r_{m}=1 \mathrm{~m}, \gamma^{*}=6.8 \mathrm{~dB}$ and path $\operatorname{loss} \alpha=2$.

obtain such ideal case only if the used bands are placed right next to each other. But, in an UNB network, the oscillator jitter causes imprecise carrier position, which can lead to an overlap between adjacent bands. Therefore, the bands should be separated by a guard interval, which must be taken into account in the spectral efficiency. We consider here a $1736 \mathrm{~Hz}$ guard band, which 
corresponds to the operating frequency transmission $868 \mathrm{MHz}$ and standard deviation of frequency jitter of $2 \mathrm{ppm}$. This permits to ensure that no actual carrier frequency would fall outside the intended band.

We choose the AR model to study this criteria while varying the path loss exponents. By observing Fig.10, we can note that for small bandwidth such as $B=12 \mathrm{kHz}$, the spectral efficiency is highly degraded by the guard band. This is due to the fact that a big portion of the band is wasted for the guard band to counteract the frequency jitter. Meanwhile, the impact of guard band diminishes as $B$ increases. Hence for the large bandwidth length, the spectral efficiency seems the same for both cases. More interestingly, for each $B$, there exists a highest spectral efficiency obtained with an optimal $\alpha$. This is because when $\alpha$ increases, the signal power of both the desired node and interfering nodes diminishes. In the first part of the curves, as the desired node is near to the BS, this decrease of power is more important for interfering nodes, which can be anywhere in the cell. Meanwhile, in the second part, when $\alpha$ exceeds a certain value, the power reduction is so severe that the desired node has no more advantage. Thus $N_{\max }$ first increases then decreases. One may note that this behavior is smoothened when $r_{x}$ is closer to $r_{M}$.

Following the previous conclusion, we have plotted in Fig. 11 the optimal bandwidth $B$ (for the guard band case) to achieve the highest ratio $\frac{N_{\max }}{B}$, as a function of the path loss exponent $\alpha$. This figure provides the optimal choice for $B$, according to the propagation characteristics. We observe that for urban areas such as $\alpha=4$, we need a thiner bandwidth than for rural areas to reach the highest spectral efficiency. Hence it also permits us to make effective use of the available bandwidth.

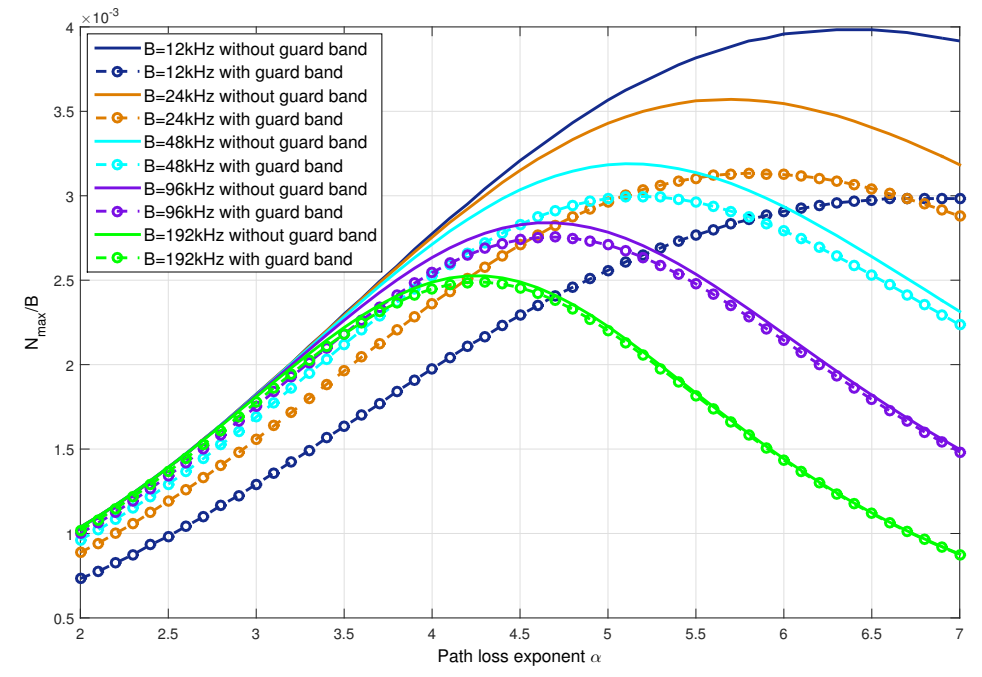

Fig. 10 Maximum node number to bandwidth ratio $\frac{N_{\max }}{B}$ (nodes/Hz) vs exponent pathloss $\alpha$, for $r_{M}=10 \mathrm{~km}, r_{x}=2 \mathrm{~km}, r_{m}=1 \mathrm{~m}, \gamma^{*}=6.8 \mathrm{~dB}$, with and without guard band 


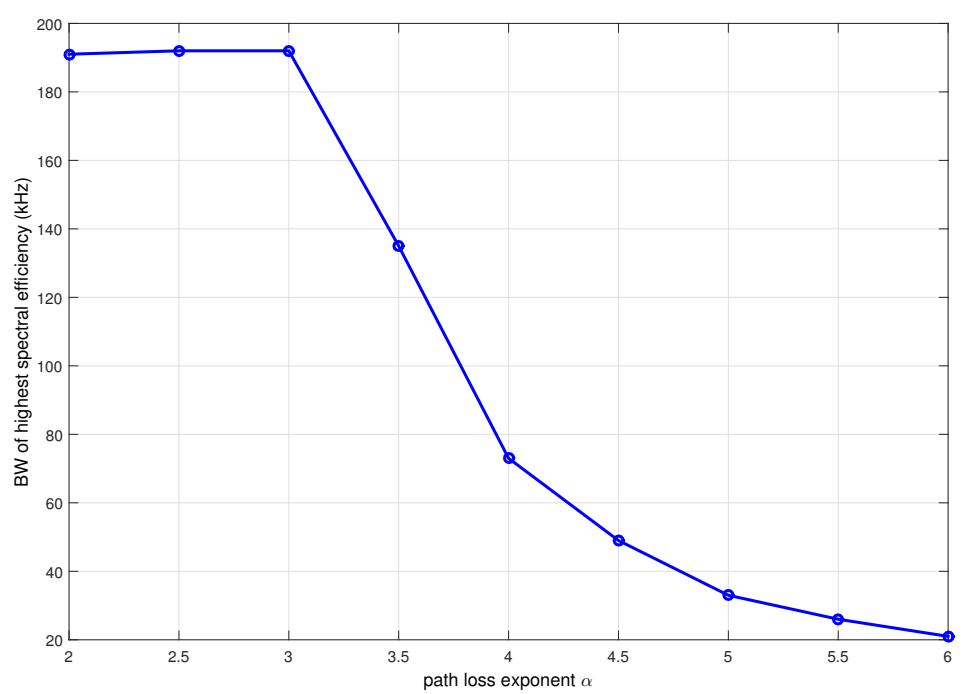

Fig. 11 Bandwidth for highest spectral efficiency $\left(\frac{N_{\max }}{B}\right)$ vs exponent path-loss $\alpha$, for $r_{M}=10 \mathrm{~km}, r_{x}=2 \mathrm{~km}, r_{m}=1 \mathrm{~m}, \gamma^{*}=6.8 \mathrm{~dB}$, with guard band

\section{Conclusion}

In this paper, we have studied the UNB network, which is a promising candidate for IoT applications. Its main specificity is the use of Random-FTMA scheme, which leads to a new behavior of the interference. We have considered two models to approximate it: Gaussian model and rectangular model. In this paper, we have studied the uplink of the UNB network, in terms of interference modeling and network capacity, when considering channel impacts (path loss and Rayleigh fading). We have theoretically derived the outage probability (OP) for two scenarios depending on the channel model: path loss only (for which the Gaussian model was used), and for both path loss and Rayleigh fading (for which the rectangular model was considered). The results showed that the OP obtained by both models conform well to the ones obtained by simulations.

Moreover, the theoretical expressions permitted us to evaluate and estimate the network capacity, in term of the maximum number of nodes for a targeted OP. We have also evaluated the spectral efficiency as a function of the pathloss exponent. We highlighted that the bandwidth which achieves the highest spectral efficiency depends on the propagation condition.

To conclude, we believe that this theoretical work is very useful for the study of UNB network capacity and its deployment. Finally, this study can be furthered by taking into account the temporal activity rate of the nodes. 
It can serve as a theoretical basis for further studies such as multiple receivers as well.

\section{References}

1. $\mathrm{Xu}, \mathrm{L} . \mathrm{D} ., \mathrm{He}, \mathrm{W} ., \mathrm{Li}, \mathrm{S}$.: Internet of things in industries: A survey. IEEE Transactions on Industrial Informatics 10(4), 2233-2243 (2014)

2. Gubbi, J., Buyya, R., Marusic, S., Palaniswami, M.: Internet of things (iot): A vision, architectural elements, and future directions. Future Generation Computer Systems 29(7), 1645-1660 (2013)

3. Goursaud, C., Gorce, J.-M.: Dedicated networks for iot: Phy/mac state of the art and challenges. EAI endorsed transactions on Internet of Things (2015)

4. http://lora-alliance.org/What-Is-LoRa/Technology, (accessed2017/01/25)

5. http://www. weightless.org/, (accessed2017/01/25)

6. http://www.sigfox.com/en/\\#//technology, (accessed2017/01/25)

7. Goursaud, C., Mo, Y.: Random unslotted time-frequency aloha: Theory and application to iot unb networks. In: 2016 23rd International Conference on Telecommunications (ICT), pp. 1-5 (2016)

8. Do, M.-T., Goursaud, C., Gorce, J.-M.: Interference modelling and analysis of random fdma scheme in ultra narrowband networks. In: AICT 2014 (2014)

9. Do, M.T., Goursaud, C., Gorce, J.M.: On the benefits of random FDMA schemes in ultra narrow band networks. In: 2014 12th International Symposium on Modeling and Optimization in Mobile, Ad Hoc, and Wireless Networks (WiOpt), pp. 672-677 (2014)

10. Haenggi, M., Andrews, J.G., Baccelli, F., Dousse, O., Franceschetti, M.: Stochastic geometry and random graphs for the analysis and design of wireless networks. IEEE Journal on Selected Areas in Communications 27(7), 1029-1046 (2009)

11. Baccelli, F., Blaszczyszyn, B., Muhlethaler, P.: An aloha protocol for multihop mobile wireless networks. IEEE Transactions on Information Theory 52(2), 421-436 (2006)

12. Nguyen, H.Q., Baccelli, F., Kofman, D.: A stochastic geometry analysis of dense IEEE 802.11 Networks. In: IEEE INFOCOM 2007 - 26th IEEE International Conference on Computer Communications, pp. 1199-1207 (2007)

13. h. Lee, C., Haenggi, M.: Interference and outage in poisson cognitive networks. IEEE Transactions on Wireless Communications 11(4), 1392-1401 (2012)

14. Sakr, A.H., Hossain, E.: Cognitive and energy harvesting-based d2d communication in cellular networks: Stochastic geometry modeling and analysis. IEEE Transactions on Communications 63(5), 1867-1880 (2015)

15. ElSawy, H., Hossain, E., Haenggi, M.: Stochastic geometry for modeling, analysis, and design of multi-tier and cognitive cellular wireless networks: A survey. IEEE Communications Surveys Tutorials 15(3), 996-1019 (2013)

16. Perrott, M.H., Salvia, J.C., Lee, F.S., Partridge, A., Mukherjee, S., Arft, C., Kim, J., Arumugam, N., Gupta, P., Tabatabaei, S., et al.: A temperature-to-digital converter for a mems-based programmable oscillator with frequency stability and integrated jitter. Solid-State Circuits, IEEE Journal of 48(1), 276-291 (2013)

17. Lim, J., Kim, H., Jackson, T.N., Choi, K., Kenny, D.: An ultra-compact and low-power oven-controlled crystal oscillator design for precision timing applications. Ultrasonics, Ferroelectrics, and Frequency Control, IEEE Transactions on 57(9), 1906-1914 (2010)

18. Walker H. R, UNB. http://www.vmsk.org

19. Xuping, Z., He, L., Haigen, H., Guoxin, Z.: Typical unb modulation methods and their spectrums. In: Wireless Mobile and Computing (CCWMC 2009), IET International Communication Conference On, pp. 283-286 (2009). IET

20. Artigue, C.: Method for searching for a useful signal in a multiplexing band. US Patent $20,150,155,960(2015)$

21. Haenggi, M., Ganti, R.K.: Interference in Large Wireless Networks. Now Publishers Inc, ??? (2009)

22. François, B., Bartłomiej, B.: Stochastic Geometry and Wireless Networks: Volume I Theory. NoW Publishers (2009) 
23. Lee, J., Tepedelenlioglu, C.: Stochastic ordering of interference in large-scale wireless networks. Signal Processing, IEEE Transactions on 62(3), 729-740 (2014)

24. Baccelli, F., Błaszczyszyn, B., Mühlethaler, P.: Stochastic analysis of spatial and opportunistic aloha. Selected Areas in Communications, IEEE Journal on 27(7), 1105-1119 (2009)

25. Haenggi, M.: On distances in uniformly random networks. IEEE Transactions on Information Theory 51(10), 3584-3586 (2005)

26. Goseling, J., Gastpar, M., Weber, J.H.: Line and lattice networks under deterministic interference models. Information Theory, IEEE Transactions on 57(5), 3080-3099 (2011)

27. Bharucha, Z., Haas, H.: The distribution of path losses for uniformly distributed nodes in a circle. Research Letters in Communications 2008, 4 (2008)

28. Kountouris, M., Pappas, N.: Approximating the interference distribution in large wireless networks. In: Wireless Communications Systems (ISWCS), 2014 11th International Symposium On, pp. 80-84 (2014). IEEE

29. Aljuaid, M., Yanikomeroglu, H.: Investigating the gaussian convergence of the distribution of the aggregate interference power in large wireless networks. Vehicular Technology, IEEE Transactions on 59(9), 4418-4424 (2010)

30. Inaltekin, H., Hanly, S.V.: On the rates of convergence of the wireless multi-access interference distribution to the normal distribution. In: Modeling and Optimization in Mobile, Ad Hoc and Wireless Networks (WiOpt), 2010 Proceedings of the 8th International Symposium On, pp. 453-458 (2010). IEEE

31. Inaltekin, H.: Gaussian approximation for the wireless multi-access interference distribution. Signal Processing, IEEE Transactions on 60(11), 6114-6120 (2012)

32. Aljuaid, M., Yanikomeroglu, H.: A cumulant-based characterization of the aggregate interference power in wireless networks. In: Vehicular Technology Conference (VTC 2010-Spring), 2010 IEEE 71st, pp. 1-5 (2010). IEEE

33. Huang, P., Xiao, L., Soltani, S., Mutka, M.W., Xi, N.: The evolution of mac protocols in wireless sensor networks: A survey. Communications Surveys \& Tutorials, IEEE 15(1), 101-120 (2013)

34. Bachir, A., Dohler, M., Watteyne, T., Leung, K.K.: Mac essentials for wireless sensor networks. Communications Surveys \& Tutorials, IEEE 12(2), 222-248 (2010)

35. Le, H.K., Henriksson, D., Abdelzaher, T.: A practical multi-channel media access control protocol for wireless sensor networks. In: Proceedings of the 7th International Conference on Information Processing in Sensor Networks, pp. 70-81 (2008). IEEE Computer Society

36. Wu, Y., Stankovic, J.A., He, T., Lin, S.: Realistic and efficient multi-channel communications in wireless sensor networks. In: INFOCOM 2008. The 27th Conference on Computer Communications. IEEE (2008). IEEE

37. Yu, Q., Chen, J., Fan, Y., Shen, X.S., Sun, Y.: Multi-channel assignment in wireless sensor networks: A game theoretic approach. In: INFOCOM, 2010 Proceedings IEEE, pp. 1-9 (2010). IEEE

38. Abramson, N.: Multiple access in wireless digital networks. Proceedings of the IEEE 82(9), 1360-1370 (1994)

39. Gorce, J.-M., Tsilimantos, D., Ferrand, P., Poor, H.V.: Energy-capacity trade-off bounds in a downlink typical cell. In: Proc. IEEE 25th International Symposium on Personal, Indoor and Mobile Radio Communications (PIMRC) (2014)

40. Hamida, E.B., Chelius, G., Gorce, J.-M.: Scalable versus accurate physical layer modeling in wireless network simulations. In: Proceedings of the 22nd Workshop on Principles of Advanced and Distributed Simulation, pp. 127-134 (2008). IEEE Computer Society

41. Baszczyszyn, B., Karray, M.K., Keeler, H.P.: Using poisson processes to model lattice cellular networks. In: INFOCOM, 2013 Proceedings IEEE, pp. 773-781 (2013)

42. Mo, Y., Goursaud, C., Gorce, J.M.: Theoretical analysis of unb-based iot networks with path loss and random spectrum access. In: 2016 IEEE 27th Annual International Symposium on Personal, Indoor, and Mobile Radio Communications (PIMRC), pp. 1-6 (2016)

43. Andrews, J.G., Baccelli, F., Ganti, R.K.: A tractable approach to coverage and rate in cellular networks. Communications, IEEE Transactions on 59(11), 3122-3134 (2011) 


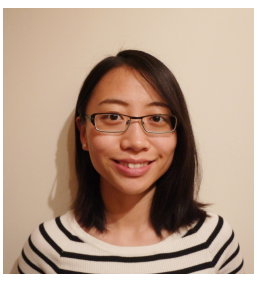

Yuqi MO received the bachelor degree in Telecommunications from Beijing Jiaotong University, China, in 2014, and the engineer diploma in Telecommunications from the National Institute of Applied Sciences (INSA), Lyon, France, in 2015, thanks to a double diplomas project between these two universities. Since Oct. 2015, she joined the CITI labs of INSA Lyon as a PhD student, as well as SigFox company as an employee. Her current research interests include LPWAN technologies for IoT networks (namely the ultra-narrow-band), radio access, physical and MAC layer design, etc.

Minh Tien DO received the B.Eng. degree in elec-

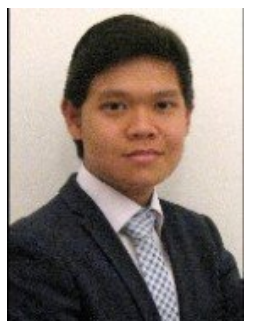
tronic and telecommunication from Bach Khoa University, Ho Chi Minh city, Vietnam, in 2010 and the M.S degree in telecommunication system from Rennes 1 University, Rennes, France, in 2011 and Ph.D degrees in Telecommunications from INSA Lyon, Lyon, France, in 2015. His research interests are mainly in the radiocommunications with current focus on mobile networks, Ultra-narrowband networks dedicated to IoTs applications, QoS measurements.

Claire Goursaud (Associate Professor INSA Lyon,


CITI) obtained her PhD in High Frequency and Optical Telecommunications in 2006 from the University of Limoges, working on Signal Processing for Optical Communications. In September 2007, she joined the INSA de Lyon, as an Assistant Professor in the telecommunication department, and the CITI laboratory. She is a specialist of coding and PHY/MAC layer design and characterization. Her current research focuses on BAN and IoT systems. She has published over 80 refereed journal and conference

papers.

Jean-Marie Gorce $[\mathrm{M} 07, \mathrm{SM} 14]$ is a member of the scientific committee of the joint lab INRIANokia Bell Labs. He received his $\mathrm{Ph} . \mathrm{D}$. degree in electrical engineering from the National Institute of Applied Sciences (INSA), Lyon, France, in 1998. He held a research position at Bracco Research, S.A. Geneva, and was recruited by INSA Lyon in 1999. He was a co-founder of the Centre for Innovation in Telecommunications and Integration of Services Laboratory of which he was the director from 2009 to 2014. He has been a researcher associated with INRIA since 2003 andhe was visiting scholar at Princeton University from September 2013 to August 2014. He is the director of the Telecommunications Department of INSA Lyon and the holder of the SPIE 
ICS Industrial Chair on the Internet of Things. He has been the principal investigator of several research projects sponsored by the French government or the European Union. He is an Associate Editor of the EURASIP journal of Wireless Communications and Networking (Springer). His research interests lie in wireless networking, focusing on realistic modeling, wireless system optimization, and performance assessment considering both infrastructure-based and ad hoc networks. He is a scientific coordinator of the experimental facility FIT-CorteXlab. 